\title{
Proyecto de intervención educativa sobre prevención del embarazo a temprana edad
}

\author{
Educational Intervention Project on Prevention of Early Pregnancy with \\ Young People
}

Projeto de intervenção educativa na prevenção da gravidez precoce

\author{
Dafne Bastida Izaguirre \\ Universidad Pedagógica Nacional 141, México \\ dafne.bastida@gmail.com \\ https://orcid.org/0000-0001-7846-7047 \\ Irma Alejandra Martínez Galván \\ Universidad Pedagógica Nacional 141, México \\ ialemartinezg@gmail.com \\ https://orcid.org/0000-0002-7778-1621 \\ Krystel Denise Ramos López \\ Universidad Pedagógica Nacional 141, México \\ krystel_ramos@hotmail.com \\ https://orcid.org/0000-0003-4532-8125 \\ Luis Alberto Ríos Dueñas \\ Universidad Pedagógica Nacional 141, México \\ albertorios.2417@gmail.com \\ https://orcid.org/0000-0001-5928-021X
}




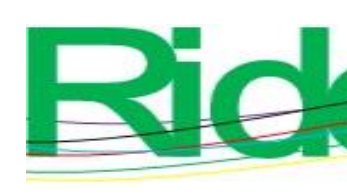

Revista Iberoamericana para la Investigación y el Desarrollo Educativo ISSN $2007-7467$

\section{Resumen}

Este trabajo presenta un proyecto de intervención que desarrollaron alumnos de cuarto grado de la licenciatura en Intervención Educativa de la Universidad Pedagógica Nacional 141. El objetivo del proyecto fue diseñar e implementar un taller que capacite a las y los adolescentes de tercer grado de la Escuela Secundaria Sor Juana Inés sobre las causas y consecuencias del embarazo a temprana edad, con la finalidad de crear un entorno que favorezca las decisiones libres, responsables e informadas sobre el ejercicio de su sexualidad. Metodológicamente, en primer lugar, se realizó un diagnóstico mediante encuesta para determinar los conocimientos previos de los alumnos. Así, más tarde, el taller contempló los temas propuestos por el alumnado y las omisiones del plan de estudios en materia de sexualidad. Las actividades dentro del taller incluyeron videos, exposiciones y juegos vivenciales que condujeran a la reflexión y tuvo una duración aproximada de tres horas. Los resultados demostraron la necesidad de ejecutar este tipo de proyectos de intervención, ya que la información que poseen los adolescentes es incompleta e inadecuada. Es fundamental que conozcan las formas de prevención para que tomen mejores decisiones y ejerzan una sexualidad de forma responsable. También es importante reconocer que este tipo de proyectos en contextos reales promueven el uso de los conocimientos y habilidades adquiridos por los estudiantes a lo largo de su trayecto educativo.

Palabras clave: competencias, educación secundaria, embarazo adolescente, intervención educativa, sexualidad.

\section{Abstract}

This work presents an intervention project developed by fourth-grade students of the bachelor's degree in Educational Intervention at the Universidad Pedagógica Nacional 141. The objective of the project was to design and implement a workshop that trains third-grade adolescents at Escuela Secundaria Sor Juan Inés de la Cruz on the causes and consequences of early pregnancy, in order to create an environment that favors free, responsible and informed decisions about the exercise of their sexuality. Methodologically, a diagnosis was made through a survey to determine the students' prior knowledge. Thus, the workshop considered the topics proposed by the students and the omissions of the curriculum on sexuality. The activities within the workshop included videos, exhibitions and experiential games that led to reflection and lasted approximately three hours. The results demonstrated 


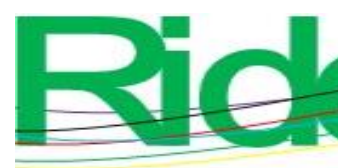

Revista Iberoamericana para la
Investigación y el Desarrollo Educativo
ISSN $2007-7467$

the need to execute this type of intervention projects, since the information that adolescents have is incomplete and inadequate. It is essential that they know the forms of prevention so that they make better decisions and have responsible sexual behavior. It is also important to recognize that these types of projects in real contexts promote the use of the knowledge and skills acquired by students throughout their educational journey.

Keywords: educational competencies, middle school, early pregnancy, educational intervention, sexuality.

\section{Resumo}

Este trabalho apresenta um projeto de intervenção desenvolvido por alunos do quarto ano do curso de Licenciatura em Intervenção Educacional da Universidade Pedagógica Nacional 141. O objetivo do projeto era conceber e implementar uma oficina de formação de adolescentes do terceiro ano do Ensino Secundário Sor Juana Inés sobre as causas e consequências da gravidez precoce, a fim de criar um ambiente que incentive decisões livres, responsáveis e informadas sobre o exercício da sexualidade. Metodologicamente, em primeiro lugar, foi feito um diagnóstico por meio de uma pesquisa para apurar os conhecimentos prévios dos alunos. Assim, posteriormente, a oficina considerou os temas propostos pelos alunos e as omissões do currículo em questões de sexualidade. As atividades da oficina incluíram vídeos, exposições e jogos vivenciais que propiciaram reflexão e duraram aproximadamente três horas. Os resultados demonstraram a necessidade de execução deste tipo de projetos de intervenção, visto que as informações de que dispõem os adolescentes são incompletas e inadequadas. É fundamental que conheçam as formas de prevenção para que possam tomar melhores decisões e exercer a sexualidade de forma responsável. É também importante reconhecer que este tipo de projetos em contextos reais promovem a utilização dos conhecimentos e competências adquiridos pelos alunos ao longo do seu percurso educativo.

Palavras-chave: competências, ensino médio, gravidez na adolescência, intervenção educativa, sexualidade.

Fecha Recepción: Marzo 2020

Fecha Aceptación: Septiembre 2020 


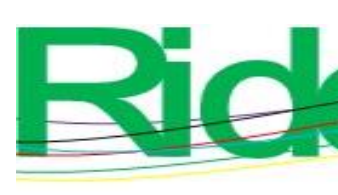

Revista Iberoamericana para la Investigación y el Desarrollo Educativo ISSN 2007-7467

\section{Introducción}

La etapa de la adolescencia está llena de cambios tanto físicos como psicológicos. En la adolescencia, la búsqueda de una identidad se intensifica. Al abandonar la niñez, y con ello cierta dependencia de los padres, los jóvenes adquieren autonomía. A partir de entonces, comienzan a formar nuevos vínculos sociales (Aberastury y Knobel, 2010; Centro Nacional de Equidad de Género y Salud Reproductiva, 2016).

Es una etapa caracterizada por distintos cambios emocionales, corporales y psicológicos, modificaciones de los roles dentro y fuera del núcleo familiar. En resumen, es un momento clave del desarrollo de un individuo (Krauskopf, 1999).

Ahora bien, es imposible hablar de la adolescencia y no hablar de sexualidad. Igualmente, al hablar de la formación en los jóvenes, la educación sexual debería ser ineludible. En múltiples documentos a nivel internacional y nacional se ha discutido la importancia de la educación sexual, así como la necesidad de que sea congruente y sustentada en información científica y que garantice la salud sexual y reproductiva de las y los adolescentes (Consejo Nacional de Población [CONAPO], 2005; Organización de las Naciones Unidas para la Educación, la Ciencia y la Cultura [UNESCO], 2010; Secretaría de Salud, 2013).

La información sobre la sexualidad en la etapa adolescente es una necesidad y tanto padres como docentes deben estar preparados cuando necesiten responder y aclarar dudas. Es necesario ahondar en varios aspectos: desde la prevención hasta la manera correcta de dar trato a los y las adolescentes que ya están en una situación como el embarazo o enfermedades de transmisión sexual.

No basta con proporcionar información institucional, sino una que esté ligada con las consecuencias que existen, siempre con fundamento en los valores esenciales para la vida cotidiana, tales como el respeto, la libertad y el autoestima, entre otras.

De acuerdo con el plan de estudios de la Secretaría de Educación Pública [SEP] (2017), en tercer grado de secundaria se lleva la materia "Formación, cívica y ética", donde se habla del embarazo adolescente, aunque la información no se extiende en todos los niveles, sino que se limita a algunas materias de curso corto. Por ejemplo, en la Escuela Secundaria Sor Juana Inés de la Cruz se imparte contenido sobre educación sexual con el apoyo de los libros de texto que suele ser orientado hacia anatomía, biología y prevención de riesgos. 


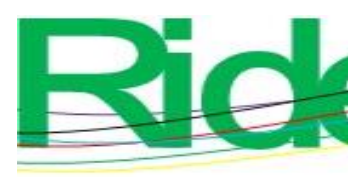

Revista Iberoamericana para la Investigación y el Desarrollo Educativo ISSN 2007 - 7467

Precisamente, una de las problemáticas que más afectan a las y los adolescentes tiene que ver con la falta de información o información fragmentada sobre sexualidad que reciben de medios o de sus amigos, y que suele traer consecuencias como un embarazo no deseado (Centro Nacional de Equidad de Género y Salud Reproductiva, 2016; CONAPO, 2005). Las estadísticas sobre embarazos en la adolescencia son llamativas tanto a nivel internacional como nacional. El Fondo de las Naciones Unidas para la Infancia (UNICEF) (Salinas, 2014) ha reportado dos millones de nacimientos a nivel mundial en menores de 15 años, de los cuales $90 \%$ se da en países en vías de desarrollo. México se posiciona en el primer lugar en embarazo adolescente entre los países de la Organización para la Cooperación y el Desarrollo Económico (OCDE) (Gobierno de la República, 2014).

Debido a esto, el Gobierno de la República anunció en el 2014 la Estrategia Nacional para la Prevención del Embarazo en Adolescentes (ENAPEA). En esta propuesta se estima que el embarazo en menores de entre 10 y 14 años ha aumentado de $1.9 \%$ en 2012 a $2.1 \%$ en 2018, mientras que el embarazo en adolescentes de entre 15 y 19 años ha disminuido de 77 nacimientos por cada 1000 a 70 nacimientos en el mismo lapso (Gobierno de la República, 2018). Esta problemática resulta de gran relevancia ya que afecta muchos aspectos del individuo, entre ellos la salud, la preparación académica, proyecto de vida, educación, aspectos económicos y sociales (Centro Nacional de Equidad de Género y Salud Reproductiva, 2016; Salinas, 2014).

Las y los adolescentes que viven esta situación llegan a sufrir rechazo, maltrato, incluso pueden continuar con patrones de pobreza y exclusión social. Entre los factores que se señalan como causas de este problema se encuentran la baja escolaridad, la falta de oportunidades debido a la situación de pobreza, trayectoria tradicional, desde las creencias religiosas hasta patrones culturales, actividad sexual precoz, legislación poco adecuada, falta de atención a la salud, violencia y matrimonios infantiles, por mencionar las más importantes (Consejo de Investigación y Evaluación de la Política Social [CIEPS], 2014; Stern, 2004).

Mirando desde perspectivas sociales y médicas, el embarazo adolescente altera la salud física, emocional, condición educativa y económica. Rangel, Valerio, Patiño, y García (2004) mencionan que se deben atender las necesidades psicosociales tanto de la adolescente embarazada como en el ámbito familiar: los cambios no solo se dan para la madre, sino también para cada integrante de la familia con la que se encuentra, las funciones que cada individuo tiene, muy posiblemente, tendrán que ser modificadas con el proceso y la llegada de este nuevo integrante, lo cual puede llegar a ser un reto para la familia. 


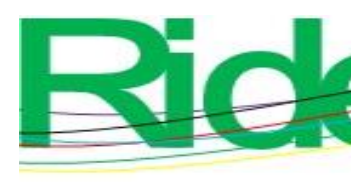

Revista Iberoamericana para la Investigación y el Desarrollo Educativo ISSN $2007-7467$

Loredo, Vargas, Casa, González y Gutiérrez (2015) añaden a la lista de consecuencias de un embarazo adolescente el rompimiento del proyecto de vida, la deserción escolar, conflictos familiares, expulsión del hogar, abandono de la pareja, alteración emocional, dificultades para la obtención de un empleo, carencia de ingresos monetarios, inclinación por el consumo de drogas y la tendencia a la prostitución o delincuencia. "Para hacer frente a este problema es necesario desarrollar estrategias preventivas orientadas al riesgo de embarazo temprano (...), mediante la implementación de programas educativos de alcance personal, familiar o escolar para este grupo etario" (Loredo et al., 2015, p. 223).

Abordar los derechos sexuales y reproductivos de los jóvenes para que tomen decisiones de forma libre y fundamentada, una legislación más rigurosa en cuanto a abuso sexual y violación, el acceso a métodos anticonceptivos de forma gratuita y mejorar los servicios de salud son algunas de las estrategias que se han propuestos desde distintos niveles (CIEPS, 2014; Gobierno de la República, 2014).

La salud del adolescente es una prioridad internacional y se aborda desde las políticas públicas. Es necesario atender la educación sexual, no solo desde una visión biológica, sino también desde una visión sociocultural, ya que hay comportamientos esperados que la sociedad regula. El ámbito educativo también coadyuva en la toma de decisiones, cuidado del cuerpo y para evitar riesgos en la población adolescente. Es de suma importancia que se lleven a cabo frecuentemente programas que promuevan la prevención de embarazo a temprana edad, con base en temas relacionados con la educación sexual, métodos anticonceptivos y su correcta utilización.

Para el proyecto de intervención llevado a cabo como parte de esta investigación se tomaron en cuenta opiniones tanto de padres de familia como de expertos. Pi (4 de agosto de 2016) argumenta que en México la sexualidad y la educación sexual continúan siendo un tabú. Por ejemplo, la organización de padres de familia denominada Red Familia se manifiesta en contra de la información impartida a los niños en las escuelas. Al hablar de estos temas, argumentan, se incrementan los embarazos en adolescentes, a pesar de que se les informa sobre el uso del condón.

Sin embargo, los expertos de la salud opinan lo contrario: creen que cada etapa de la vida debe disfrutarse siempre favoreciendo el bienestar social, disminuyendo las brechas de género con condiciones que aporten libertad a los proyectos de vida. En las diferentes etapas de la vida, según la Fundación Huésped (2012), se debe tener claridad e información sobre 


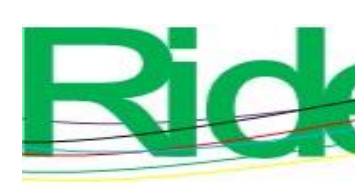

Revista Iberoamericana para la
Investigación y el Desarrollo Educativo
ISSN $2007-7467$

la salud sexual y reproductiva, para que disminuya la barrera de pobreza, discriminación, exclusión y falta de oportunidades.

En una entrevista con la Mtra. Rocío Asencio Jaime (10 de abril de 2019, comunicación personal), quien es coordinadora del programa de la Especialización en Estudios de Género de la Universidad Pedagógica Nacional Unidad 141, comenta que sí es necesario darles información a los jóvenes de tercer grado de secundaria sobre métodos anticonceptivos y la prevención del embarazo. Aún más, cree que no solo es propio de las secundarias, sino incluso se debe brindar información sobre el tema desde la primaria.

Menciona que hay mucho desconocimiento de los derechos sexuales y reproductivos de las personas jóvenes.

Los padres argumentan que ellos deciden por sus hijos, cuando también existen los derechos sexuales de las niñas, niños, las y los adolescentes, donde la información sobre ellos tendría que darles acceso a los métodos anticonceptivos. No creo que sea una parte que les toque decidir a los padres de familia, es parte del currículo educativo, en eso no tienen incidencia los padres de familia (Asencio, 10 de abril de 2019, comunicación personal).

También indicó que los adolescentes deben de tener acceso a los métodos anticonceptivos de forma gratuita en centros de salud y que sus expectativas de vida tienen influencia en sus decisiones.

Por su parte, Coleman y Hendry (1980) afirman, basados en las teorías de Piaget, que es de suma importancia el desarrollo intelectual en la etapa siguiente de la pubertad, ya que esto fundamenta la capacidad individual de acercarse a la madurez de manera que facilite su participación en la sociedad. En cualquier etapa de nuestras vidas debemos hacer valer nuestros derechos; para hacerlo necesitamos conocerlos. Siguiendo aquí a Rosales (2011), las decisiones deben estar fundamentadas con el objetivo de actuar de manera consciente e informada, teniendo en cuenta aspectos como la fecundidad, la anticoncepción, el embarazo, el aborto y la salud materna, en el caso de la salud reproductiva.

En síntesis, la educación sexual integral es esencial para los jóvenes. Esta debe ser adecuada, fundamentada y oportuna, de acuerdo con la etapa de la vida de los receptores. Y ante la negativa de algunos padres sobre la exposición de contenidos sexuales, las instituciones deben hacer el intento de presentar los temas necesarios para que los jóvenes lleven una vida sexual sana y sin riesgos. 


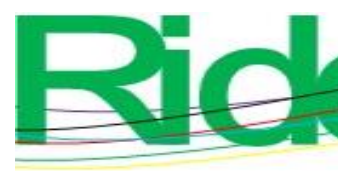

Revista Iberoamericana para la Investigación y el Desarrollo Educativo ISSN $2007-7467$

Estar informados permite la toma de decisiones fundamentadas, que los jóvenes puedan hacer valer sus derechos referentes a la sexualidad con responsabilidad, y así evitar no solo los embarazos precoces, sino también las enfermedades de transmisión sexual, entre otros aspectos.

Debido a todo lo hasta aquí comentado, se planteó la necesidad de diseñar e implementar un proyecto de intervención orientado a la prevención del embarazo a temprana edad y sus posibles consecuencias. El taller está planeado y dirigido a estudiantes de tercer grado de secundaria, aunque sus contenidos también son óptimos para los diferentes grados del nivel, ya que se revisan temas de interés en esta etapa de la vida, tal y como la prevención del embarazo, prevención de enfermedad de transmisión sexual y conocimiento y correcta utilización de métodos anticonceptivos.

\section{Objetivos del proyecto}

El objetivo general del proyecto de intervención fue diseñar e implementar un taller que garantizara a las y los adolescentes el derecho a recibir una educación sexual integral que les informe sobre las causas y consecuencias del embarazo a temprana edad, con la finalidad de crear un entorno que favorezca las decisiones libres, responsables e informadas sobre el ejercicio de su sexualidad.

De ahí se desprendieron tres objetivos específicos: 1) elaborar el diseño de un taller informativo sobre causas y consecuencias del embarazo adolescente, métodos anticonceptivos y enfermedades de transmisión sexual; 2) implementar el taller de sexualidad en la Escuela Secundaria Sor Juana Inés De La Cruz dirigido a los adolescentes de tercer grado para promover la toma de decisiones fundamentada en la información y la reflexión, y 3) sistematizar la información producto de la implementación del taller a través de la observación. 


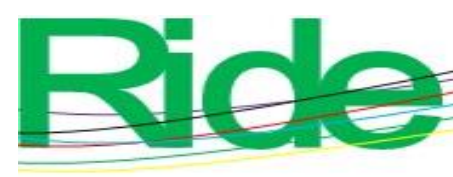

Revista Iberoamericana para la Investigación y el Desarrollo Educativo ISSN 2007-7467

\section{Método}

\section{Proyecto de intervención socioeducativa}

Este tipo de proyectos surgen como una estrategia ante la realidad social. Se trata de una serie de actividades que presentan soluciones a problemas detectados en el ámbito educativo, donde los interventores llevan a la práctica las competencias y habilidades adquiridas en su trayecto formativo. El planteamiento para este tipo de proyectos deberá tomar en cuenta las características de la población a la que se dirige, plantearse el objetivo del proyecto conforme al diagnóstico de necesidades y, con ello, hacer la propuesta de solución o diseño de proyecto, tomando en cuenta los recursos materiales y humanos disponibles para establecer las estrategias de acción (Castillo y Cabrerizo, 2011).

\section{Participantes}

El proyecto de intervención se llevó a cabo en la Secundaria Sor Juana Inés de la Cruz, ubicada en Tlajomulco de Zúñiga, Jalisco. La muestra fue no probabilística, ya que fueron seleccionados por su accesibilidad. En total, participaron 109 adolescentes de cuatro grupos de tercer grado, 48 varones y 61 mujeres.

\section{Diagnóstico de necesidades}

Se decidió realizar un diagnóstico de necesidades sobre sexualidad y uso de anticonceptivos para conocer la información con la que cuentan los estudiantes y saber cuáles son las ausencias que debemos de atender. En este caso, el diagnóstico es una herramienta indispensable y una fase en el proyecto de intervención que permite identificar la realidad que se da en el ámbito social al que vamos a acceder, que nos permitirá priorizar las acciones y, con todo ello, hacer el diseño del taller. Primero, se tuvo una entrevista con el director de la escuela, quien nos mencionó la importancia de la aplicación del taller. Entre los datos que proporcionó, es que solo hay dos casos de adolescentes embarazadas en un período de dos años. La recolección de información fue a través de una encuesta breve a los 109 estudiantes. Los resultados de la encuesta refieren que $63 \%$ de los adolescentes consideran como causas del embarazo adolescente la falta de información sobre métodos anticonceptivos, $19 \%$ dice que falta información sobre sexualidad, $11 \%$ menciona la falta de valores y $9 \%$ la ausencia de un proyecto de vida. Casi la mitad de los alumnos, $49 \%$, piensa que para obtener como 


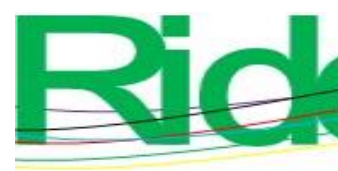

Revista Iberoamericana para la
Investigación y el Desarrollo Educativo
ISSN $2007-7467$

resultado un embarazo hace falta más de una relación sexual; es decir, tienen la idea de que no es posible que un espermatozoide fecunde un óvulo por el hecho de ser única ocasión.

Esto en contraste con $39 \%$ de los alumnos, quienes piensan que puede haber embarazo en la primera ocasión; 12 \%o supo contestar. Cabe destacar que, a pesar de la falta de información, los estudiantes consideran que las consecuencias de tener relaciones sexuales sin protección es tarea que compete a ambas partes involucradas, hombre y mujer, de manera igualitaria; es mínima la cantidad de alumnos que creen responsables a otros factores como la familia, o que la responsabilidad debe recaer sobre la adolescente embarazada.

\section{Procedimiento}

El taller propuesto titulado ;Mejor me cuido! (Vive tu vida, antes de crear una vida) fue diseñado con el propósito de informar a los y las jóvenes de tercer grado de secundaria sobre la prevención del embarazo a temprana edad y sus posibles consecuencias. Las áreas para abordar fueron los motivos que llevan a una relación sexual en adolescentes, sus consecuencias, la prevención y métodos anticonceptivos.

Con este taller se pretende proporcionar información y fomentar en ellos la responsabilidad para la toma de decisiones en materia de embarazo adolescente, que conozcan los tipos y utilización correcta de los métodos anticonceptivos, así como la importancia de estos.

Como parte del diseño curricular, fueron propuestas tres competencias que los alumnos deben desarrollar con ayuda del taller, a saber: 1) que los alumnos tengan la capacidad de autonomía y responsabilidad de sus actos a través de la adquisición de información adecuada sobre sexualidad y reproducción; 2) los alumnos deben generar conceptos propios sobre los métodos anticonceptivos, las enfermedades de transmisión sexual, así como los riesgos antes, durante y después del embarazo adolescente basados en información confiable, y 3) los alumnos deben generar actitudes positivas para una sexualidad sana que se unan a una cultura que favorezca una sexualidad libre, responsable y sin riesgo. 

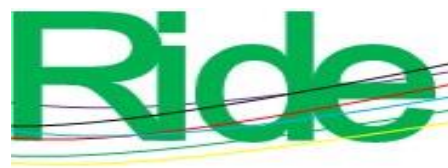

Revista Iberoamericana para la Investigación y el Desarrollo Educativo ISSN $2007-7467$

De esta manera, el taller busca informar sobre cómo sucede un embarazo y qué acciones pueden tomar para reducir el riesgo; cuáles son los diferentes métodos anticonceptivos y su uso correcto; muestra las posibles consecuencias que tiene un embarazo a temprana edad; en general, saber identificar si se encuentran en una situación de peligro y que tengan conocimiento de los lugares a donde pueden acudir por ayuda y orientación.

Después de conocer un poco de ellos, e identificar algunas de sus inquietudes o necesidades de aprendizaje sobre el tema mediante las encuestas y la observación, se elaboraron las cartas descriptivas del taller en general (tabla 1) y cada una de las actividades presentadas con los siguientes temas: "Viviendo el embarazo" (tabla 2), "Embarazo adolescente" (tabla 3) y "Métodos anticonceptivos" (tabla 4). 


\section{Presentación de las cartas descriptivas del taller}

Tabla 1. Carta descriptiva general del taller

\begin{tabular}{|c|c|}
\hline Nombre del taller & ¡Mejor me cuido! (Vive tu vida antes de crear una vida) \\
\hline $\begin{array}{l}\text { Perfil de los } \\
\text { alumnos }\end{array}$ & $\begin{array}{l}\text { Jóvenes entre } 13 \text { y } 15 \text { años de edad. Alumnos de tercer grado de } \\
\text { secundaria. Mayoritariamente, mujeres. }\end{array}$ \\
\hline $\begin{array}{l}\text { Requisitos para } \\
\text { el profesor }\end{array}$ & $\begin{array}{l}\text { Conocimiento teórico sobre temas de derechos sexuales y } \\
\text { reproductivos, prevención del embarazo, métodos anticonceptivos, } \\
\text { enfermedades de transmisión sexual y prevención de todos los } \\
\text { anteriores. }\end{array}$ \\
\hline $\begin{array}{l}\text { Campo } \\
\text { formativo }\end{array}$ & Exploración y conocimiento de la sexualidad. \\
\hline Competencia & $\begin{array}{l}\text { Conoce y practica hábitos para el cuidado de su cuerpo, prevención } \\
\text { del embarazo adolescente y enfermedades. }\end{array}$ \\
\hline Técnica & Refuerzo o ampliación. \\
\hline $\begin{array}{l}\text { Teoría educativa } \\
\text { que lo sustenta }\end{array}$ & $\begin{array}{l}\text { - Teoría sociocultural de Vygotsky. } \\
\text { - El adolescente y la libertad de Arminda Aberastury. } \\
\text { - Pensar y razonar. En Psicología de la adolescencia de John } \\
\text { Coleman y L. B. Henry. } \\
\text { - Derechos sexuales y reproductivos de Adriana Leona } \\
\text { Rosales. }\end{array}$ \\
\hline Duración & 3 horas \\
\hline $\begin{array}{l}\text { Conocimientos } \\
\text { previos }\end{array}$ & $\begin{array}{l}\text { Desarrollo del adolescente tanto cognitivo como a nivel biológico, } \\
\text { físico y social; causas y consecuencias de una vida sexual activa en la } \\
\text { pubertad y adolescencia. }\end{array}$ \\
\hline Instrucciones & $\begin{array}{l}\text { - Definir los materiales a utilizar. } \\
\text { - Recolección de métodos anticonceptivos para demostración. } \\
\text { - Dinámica de presentación (15 min). } \\
\text { - Encuesta de conocimientos previos (10 min). } \\
\text { - Actividad: "Simulador de embarazo" (20 min). } \\
\text { - Demostración de video: "Todo a su tiempo" (15 min). } \\
\text { - Exposición del tema: "Causas y consecuencias del embarazo } \\
\text { - } \text { adolescente" (20 min). } \\
\text { - Temal de mensajes (20 min). } \\
\text { - Actividad: "Demostración de métodos anticonceptivos" (30 } \\
\text { - Evaluación (cuestionario) (10 min). } \\
\text { - Cierre del taller (Colectivo Ollin) (20 min). }\end{array}$ \\
\hline
\end{tabular}




\begin{tabular}{|l|l|}
\hline Material & $\begin{array}{l}\text { } \text { didáctico } \\
\text { - }\end{array}$ \\
& Carteles \\
& - Simuetas para identificación \\
& - Tecnologías de la información \\
& - Métodos anticonceptivos \\
& - Cuípticos informativos \\
\hline Espacio & Aula de la secundaria Sor Juana Inés de la Cruz \\
\hline Evaluación & $\begin{array}{l}\text { Uso de técnicas para obtener información que posibilite conocer el } \\
\text { nivel de conocimiento que los y las adolescentes tienen sobre las } \\
\text { relaciones sexuales y la prevención del embarazo. ¿Cuál es su } \\
\text { experiencia, valores, participación y responsabilidades sobre la } \\
\text { educación referente al cuidado de la sexualidad por medio de las } \\
\text { actividades y cuestionarios? }\end{array}$ \\
\hline
\end{tabular}

Fuente: Elaboración propia

Tabla 2. Carta descriptiva de la actividad uno

\begin{tabular}{|c|c|}
\hline $\begin{array}{l}\text { Nombre de la } \\
\text { actividad }\end{array}$ & Viviendo el embarazo \\
\hline $\begin{array}{l}\text { Perfil de los } \\
\text { alumnos }\end{array}$ & $\begin{array}{l}\text { Jóvenes entre } 13 \text { y } 15 \text { años de edad. Alumnos de tercer grado de } \\
\text { secundaria. Mayoritariamente, mujeres. }\end{array}$ \\
\hline $\begin{array}{l}\text { Requisitos para el } \\
\text { profesor }\end{array}$ & $\begin{array}{l}\text { Conocimiento teórico sobre temas del embarazo adolescente, } \\
\text { cuidado del cuerpo humano y sexualidad con causas y consecuencias. }\end{array}$ \\
\hline Campo formativo & Desarrollo de la natalidad. \\
\hline Competencia & Conoce y experimenta cómo se vive un embarazo. \\
\hline Técnica & Observación de las etapas del embarazo. \\
\hline Duración & 3 horas \\
\hline $\begin{array}{l}\text { Conocimientos } \\
\text { previos }\end{array}$ & Etapas del embarazo, cuidados médicos, alimentaciór \\
\hline Instrucciones & $\begin{array}{l}\text { - } \text { Recolección del material. } \\
\text { - Voluntarios para la actividad (hombre y mujer). } \\
\text { - Colocación del simulador de embarazo. } \\
\text { - Llevar a cabo las etapas del aumento de peso durante el } \\
\text { - } \text { embarazo. } \\
\text { - Testimonio de los participantes. }\end{array}$ \\
\hline $\begin{array}{l}\text { Material } \\
\text { didáctico }\end{array}$ & $\begin{array}{ll}\text { - } & \text { Simulador de embarazo } \\
\text { - } & 6 \text { bolsas de arroz de medio kilo cada una }\end{array}$ \\
\hline Espacio & Aula de la secundaria Sor Juana Inés de la Cruz \\
\hline Evaluación & $\begin{array}{l}\text { Conocimiento de las etapas del embarazo, actitud ante las } \\
\text { dificultades. ¿Cuál es su experiencia, valores, participación y } \\
\text { responsabilidades sobre la educación referente al cuidado de la salud } \\
\text { por medio de la actividad? }\end{array}$ \\
\hline
\end{tabular}

Fuente: Elaboración propia 
Tabla 3. Carta descriptiva de la actividad dos

\begin{tabular}{|c|c|}
\hline $\begin{array}{lll}\begin{array}{l}\text { Nombre de la } \\
\text { actividad }\end{array} & \\
\end{array}$ & Mural de mensajes \\
\hline $\begin{array}{l}\text { Perfil de los } \\
\text { alumnos }\end{array}$ & $\begin{array}{l}\text { Jóvenes entre } 13 \text { y } 15 \text { años de edad. Alumnos de tercer grado de } \\
\text { secundaria. Mayoritariamente, mujeres. }\end{array}$ \\
\hline $\begin{array}{l}\text { Requisitos para } \\
\text { el profesor }\end{array}$ & $\begin{array}{l}\text { Conocimiento teórico sobre temas del embarazo adolescente, cuidado } \\
\text { del cuerpo humano y sexualidad con causas y consecuencias. }\end{array}$ \\
\hline $\begin{array}{l}\text { Campo } \\
\text { formativo }\end{array}$ & Desarrollo de la natalidad. \\
\hline Competencia & Conoce y experimenta cómo se vive un emba \\
\hline Técnica & Observación y reconocimiento de palabras. \\
\hline Duración & 20 minutos \\
\hline $\begin{array}{l}\text { Conocimientos } \\
\text { previos }\end{array}$ & Identificación de emociones, conceptos y visualización de palabras. \\
\hline Instrucciones & $\begin{array}{l}\text { - } \text { Recolección del material. } \\
\text { - Entrega de palabras para identificar. } \\
\text { - Leer en voz alta y pasar al pizarrón a pegar en su casilla } \\
\text { correspondiente. } \\
\text { - Testimonio de los participantes. }\end{array}$ \\
\hline $\begin{array}{l}\text { Material } \\
\text { didáctico }\end{array}$ & $\begin{array}{ll}\text { - } & \text { Pósits de colores } \\
\text { - } & \text { Plumas } \\
\text { - } & \text { Pizarrón con casillas identificadas }\end{array}$ \\
\hline Espacio & Aula de la secundaria Sor Juana Inés de la Cruz \\
\hline Evaluación & $\begin{array}{l}\text { Conocimiento de los significados de diversas palabras relacionadas } \\
\text { con la sexualidad. ¿Cuál es su experiencia, valores, participación y } \\
\text { responsabilidades sobre la información referente a la sexualidad por } \\
\text { medio de la actividad? }\end{array}$ \\
\hline
\end{tabular}

Fuente: Elaboración propia 
Tabla 4. Carta descriptiva de la actividad tres

\begin{tabular}{|c|c|}
\hline $\begin{array}{l}\text { Nombre de la } \\
\text { actividad }\end{array}$ & Demostración de métodos anticonceptivos \\
\hline $\begin{array}{l}\text { Perfil de los } \\
\text { alumnos }\end{array}$ & $\begin{array}{l}\text { Jóvenes entre } 13 \text { y } 15 \text { años de edad. Alumnos de tercer grado de } \\
\text { secundaria. Mayoritariamente, mujeres. }\end{array}$ \\
\hline $\begin{array}{l}\text { Requisitos para } \\
\text { el profesor }\end{array}$ & $\begin{array}{l}\text { Conocimiento teórico sobre los métodos anticonceptivos y la manera } \\
\text { correcta de utilizarlos. }\end{array}$ \\
\hline $\begin{array}{l}\text { Campo } \\
\text { formativo }\end{array}$ & Desarrollo de habilidades técnicas. \\
\hline Competencia & Conoce y experimenta cómo se utiliza un método anticonceptivo. \\
\hline Técnica & Práctica participativa. \\
\hline Duración & 30 minutos \\
\hline $\begin{array}{l}\text { Conocimientos } \\
\text { previos }\end{array}$ & Uso y funcionalidad de métodos \\
\hline Instrucciones & $\begin{array}{l}\text { - Recolección del material. } \\
\text { - } \text { Demostración de cómo se utilizan los métodos anticonceptivos. } \\
\text { - Participación de algunos alumnos. } \\
\text { - Testimonio de los participantes. }\end{array}$ \\
\hline $\begin{array}{l}\text { Material } \\
\text { didáctico }\end{array}$ & $\begin{array}{l}\text { - Pene y vagina de látex } \\
\text { - Condones femeninos y masculinos } \\
\text { - Trípticos } \\
\text { - Juego con imágenes de los anticonceptivos }\end{array}$ \\
\hline Espacio & Aula de la secundaria Sor Juana Inés de la Cruz \\
\hline Evaluación & $\begin{array}{l}\text { Conocimiento de los métodos anticonceptivos, la utilización correcta } \\
\text { de los mismos. ¿Cuál es su experiencia de la actividad, valores y } \\
\text { participación en la actividad? }\end{array}$ \\
\hline
\end{tabular}

Fuente: Elaboración propia

\section{Implementación del taller}

Se llevó a cabo del 6 al 9 de mayo de 2019; se destinó un día a cada grupo. Se hizo un registro sobre la forma de trabajo de cada grupo y una evaluación final donde se les preguntó si el taller fue de su interés. Los resultados fueron registrados por medio de observación participante por parte de los expositores.

\section{Resultados de la experiencia por grupo}

La aplicación del taller se llevó a cabo en el interior de los salones con el apoyo del equipo audiovisual proporcionado por la dirección de la secundaria. Vale igualmente la pena destacar que contamos con amplia disposición de los profesores para destinar tiempo de sus clases a la realización de las actividades, las cuales fueron cumplidas con la continuidad y tiempo asignados inicialmente. 


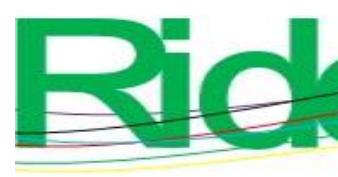

Revista Iberoamericana para la
Investigación y el Desarrollo Educativo
ISSN $2007-7467$

En un contexto general, el proceso fue efectuado con base en la metodología, la organización, los procedimientos y las actividades utilizando los instrumentos y materiales didácticos de acuerdo con la estrategia de acción.

\section{Grupo 3. ${ }^{\circ} \mathrm{A}$}

El lunes 6 de mayo del 2019 se trabajó con el grupo 3. A. La reacción de los alumnos al principio fue de expectativa. La participación fue constante durante todo el taller y mostraron interés en el tema "Prevención del embarazo"; sin embargo, el momento más activo de todo el taller fue la demostración del uso correcto de métodos anticonceptivos. Durante esta, se alentaban a participar entre ellos y expresaban comentarios y risas por lo gráfico de los materiales. La actividad fue una prueba del desconocimiento del uso correcto en la práctica, sin embargo, los participantes se brindaron apoyo, recordando la información que acababan de recibir, y lograron colocar de manera correcta los diferentes métodos anticonceptivos en el material didáctico.

Del mismo modo, durante el "Mural de mensajes" se observó que tienen una vaga idea sobre la existencia de diferentes métodos anticonceptivos. En su mayoría, no sabían cuáles son ni la forma de utilizarlos. Casi en todas las notas se leía "Conozco el condón”, y en unas cuantas, "La operación como mi mamá” o "Mi mamá tiene el DIU”.

Con la actividad "Simulador de embarazo" se logró que los adolescentes reflexionaran sobre las dificultades que pasan cuando se convierten en padres a temprana edad. En el testimonio final, expresaron que no les gustaría ser papá y mamá joven. Algunos comentarios fueron como "papás": "Es muy enfadoso estar atendiendo a la embarazada"; y como "mamás": "Pesa mucho la panza, es incómoda". Para experimentar la sensación, algunas alumnas quisieron colocarse el simulador para "ver qué se siente" e hicieron comentarios sobre el peso y la incomodidad.

En este grupo tenían un conocimiento previo limitado; después del taller, de acuerdo con la retroalimentación de los alumnos, se aumentó su entendimiento, así como el interés sobre el tema. 

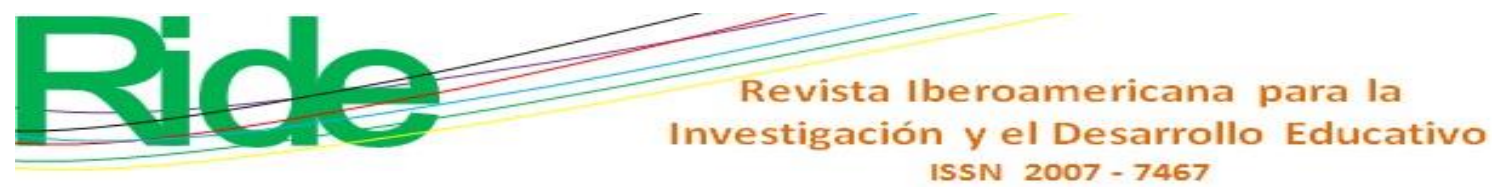

\section{Grupo 3. ${ }^{\circ} \mathrm{C}$}

El martes 7 de mayo del 2019 se trabajó con el grupo C. En este caso, se notó poca participación y experimentamos apatía por parte de los adolescentes durante todo el taller. La demostración de métodos anticonceptivos fue tomada con menor seriedad y nos costó un poco más de trabajo mantenerlos enfocados en la información. Cabe destacar que fue el único grupo en el que la profesora estuvo presente durante el taller, lo cual aumentó la timidez de los alumnos y mermó la participación, debido a que ella les dirigía preguntas y comentarios sobre el tema, lo que ocasionó un ambiente de tensión y seriedad.

Durante la presentación de las fichas técnicas descriptivas de los métodos anticonceptivos, hubo comentarios como: “Ay! Yo no sabía que existía eso”. Una de las estudiantes compartió que ella había acudido en busca de información al centro de salud de su localidad y la persona que le atendió le hizo el comentario siguiente: "Niña, estás muy chiquita para preguntar eso, ¿tu mamá sabe que andas buscando esas cosas?”, lo que denota que las instituciones públicas cuentan con poca capacitación del personal que labora en ellas. Sobre la actividad "Simulador de embarazo", el testimonio de los alumnos fue, en el caso de una "mamá": "Con la panza no quepo en las bancas y no me gusta estarme cuidando tanto"; en el caso de un "papá": "No me gusta tener que estar ayudando, qué enfadoso".

\section{Grupo 3. ${ }^{\circ}$ B}

El miércoles 8 de mayo del 2019 se aplicó la intervención al grupo B. Aquí se obtuvo una participación sumamente activa, con respeto, seriedad y todo el tiempo mostraron un interés genuino. Durante la actividad "Mural de mensajes", se demostró que en dicho grupo los conocimientos sobre la variedad existente de métodos anticonceptivos eran más amplios y con mayor diversidad. Hubo dos alumnas que se describieron a sí mismas como demasiado apenadas todo el tiempo, incluso una de sus compañeras mencionó que "ella nunca quiere hablar de sexo, le da vergüenza"; a pesar de esto, la atención y participación general del grupo fue favorable y con mucha disposición de aprender. Constantemente se interrumpían las exposiciones o actividades para la resolución de dudas emergentes en las que algunos hacían notas y comentaban entre sí.

Durante la demostración, se hizo notar la atención que prestaron durante la exposición de la teoría, participaron en la colocación y mencionaron de manera conjunta los procedimientos y maneras de utilización expuestas con anterioridad. 


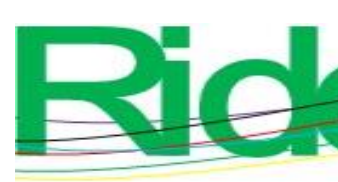

Revista Iberoamericana para la
Investigación y el Desarrollo Educativo
ISSN $2007-7467$

información que consideran puede ser de utilidad en la vida cotidiana y les ayudará a tener una perspectiva objetiva para futuras decisiones. El taller, sus actividades y contenidos fueron calificados de forma general como satisfactorios e interesantes. En este cuestionario también se manifestó un especial interés en la necesidad de recibir este tipo de talleres y el abordaje de otros temas de importancia como las drogas, el aborto, educación ambiental y depresión, mientras en todos los grupos nos reiteraban lo mucho que les había gustado la dinámica.

\section{Discusión}

Los resultados de la aplicación del taller reflejan un desconocimiento del tema y la necesidad de abordar con las y los adolescentes las causas y consecuencias del embarazo a temprana edad y los métodos que se pueden utilizar para evitarlo. Otros proyectos de intervención que se han realizado con la misma temática resaltan la importancia de implementar estos talleres en la escuela, ya que es un espacio propicio para abordar acciones de prevención con los jóvenes. La información les brinda la oportunidad de tomar decisiones responsables (Rodrigues et al., 2010).

Se hace conciencia de la necesidad que tienen los jóvenes mexicanos de aprender y resolver las dudas que les aquejan en la vida cotidiana, sobre todo los que se encuentran en desventajas sociales. Causa extrañeza que, a pesar del uso y acceso a la información en esta época, aún exista una cultura de negación o prohibición por parte de algunos padres de familia, que lejos de cimentar las bases para que sus hijos sean sexualmente sanos prefieren ignorar que la sexualidad de los adolescentes existe, omitiendo su responsabilidad y dejando tan importante tarea a las diversas fuentes que pueden no ser las más adecuadas (Herrera et al., 2018).

Rodrigues et al. (2010) mencionan que la escuela debe ser un espacio donde los adolescentes se informen y aclaren sus dudas para de esta manera sensibilizarlos y que opten por decisiones conscientes y responsables. Sin embargo, algunos docentes, por miedo a las reacciones de los padres de familia, por desconocimiento o incomodidad, prefieren evitar esos temas.

¿Cómo podrían los adolescentes hacer valer sus derechos sexuales y reproductivos cuando en primera instancia no los conocen? 


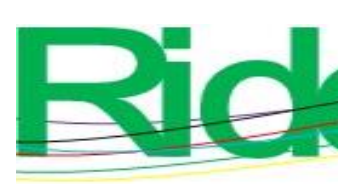

Revista Iberoamericana para la Investigación y el Desarrollo Educativo ISSN $2007-7467$

Es importante capacitar a los docentes en temas de sexualidad para que puedan atender esta dimensión tan importante en la vida de los adolescentes. Aunque también en esta parte se involucran otras cuestiones de índole personal que pueden limitar a los profesores a compartir información libre de prejuicios y estereotipos, basada simplemente en evidencia científica y en los derechos humanos (Medina, 2017).

Por otro lado, la educación sexual se puede complementar en atención a la sociedad por medio de los interventores educativos, quienes en otros espacios son llamados también como educadores sociales. A través de la educación no formal, pueden apoyar en estos procesos educativos, tomando en cuenta sobre todo que saben establecer lazos de confianza para que los estudiantes se expresen y que son expertos en el diseño de proyectos de intervención por su manera de entender la complejidad de los procesos que se dan en el ámbito socioeducativo (Carrillo y Cabrerizo, 2011).

Entre las limitaciones del trabajo se considera que un solo taller no es suficiente para que los alumnos tengan la información pertinente que los conduzca a tomar las decisiones adecuadas. Aun así, no está de más volverlo a mencionar, se logró sensibilizar a los participantes sobre la problemática. El trabajo se enfocó en un solo centro escolar debido a que el equipo de facilitadores es pequeño, pero es importante que se pueda replicar en otros espacios para ver la respuesta de las y los adolescentes.

Entre las fortalezas del estudio, por su parte, podemos mencionar el apoyo y la participación de todos los docentes de la licenciatura para el desarrollo del trabajo. Cada uno de ellos aportó su conocimiento en el área que se requería para llevar a cabo el proyecto. Igual de valioso fue el apoyo de las autoridades de la secundaria, donde a veces es difícil abordar estos temas ante la presión de padres de familia y la omisión de los docentes, como se mencionó anteriormente.

Por último, entre las debilidades se encuentra el tiempo del taller (tres horas es poco tiempo para abordar a profundidad la temática) y los contenidos (hay temas que es necesario incorporar, por ejemplo, el conocimiento de los derechos sexuales y reproductivos para adolescentes). Los instrumentos aplicados para obtener el diagnóstico y la encuesta de impacto del proyecto fueron igualmente muy breves; se requiere un mejor diseño que aporte más información sobre lo que las y los adolescentes conocen de sexualidad y el impacto del taller. 


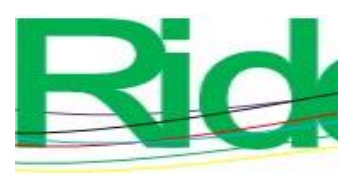

Revista Iberoamericana para la Investigación y el Desarrollo Educativo ISSN $2007-7467$

\section{Conclusiones}

Este taller demostró que bajo el contexto institucional los jóvenes aprenden lo necesario para saber cómo está conformado su cuerpo físicamente; sin embargo, aún queda un largo camino por recorrer en cuestiones educativas, las cuales deben brindar la capacidad de decidir y tomar acciones con base en mejores conocimientos que reduzcan las prácticas de riesgo. También destaca la importancia de abordar la sexualidad desde la parte lúdica con la complejidad que ello implica, evitar el enfoque reduccionista de mirar solo desde la parte biológica o darle una connotación negativa. Una siguiente propuesta en el taller sería el reconocer la importancia del abordaje de los derechos sexuales y reproductivos de las y los adolescentes y también enfatizar la dimensión del placer en la sexualidad.

Conforme a los resultados en las evaluaciones, a los comentarios posteriores de la visualización de los docentes sobre las actitudes y mejoras de sus alumnos y a todo lo observado por medio de la interacción vivida con los alumnos, rescatamos que este proyecto ha sido beneficioso y abre un mar de oportunidades para la investigación, diseño, implementación de proyectos de intervención que fomenten la toma de decisiones responsable y, sobre todo, a que se considere la distribución y exposición de contenidos adecuados y oportunos de acuerdo con cada nivel escolar, tomando en cuenta las necesidades de la comunidad con la que se trabaja.

Se considera como línea de investigación a futuro el trabajo con docentes para capacitarlos en torno al manejo de la sexualidad integral en adolescentes y un análisis y evaluación de las políticas públicas orientadas a la prevención del embarazo adolescente que en realidad promuevan la educación integral en sexualidad y la construcción del proyecto de vida, con la finalidad de que asuman la responsabilidad de sus decisiones.

En cuanto al proyecto en general, se considera que este tipo de trabajos como parte del currículo escolar son necesarios, porque los conocimientos y habilidades adquiridos durante los cursos se demuestran durante el desarrollo y la implementación de la intervención en un contexto real. Esta estrategia de aprendizaje por proyectos promovió el uso de las competencias adquiridas hasta el momento durante la licenciatura, estimuló la investigación y el pensamiento crítico de los estudiantes al generar en ellos propuestas de solución a problemas reales. 


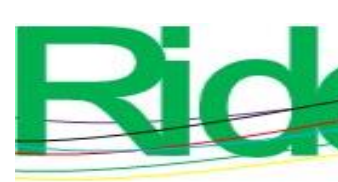

Revista Iberoamericana para la
Investigación y el Desarrollo Educativo
ISSN $2007-7467$

\section{Agradecimientos}

A los directivos de la Escuela Secundaria Sor Juana Inés de la Cruz y de la Universidad Pedagógica Nacional 141 por su apoyo en la realización de este proyecto. Al Consejo Nacional de Ciencia y Tecnología (CONACYT) por la aportación de recursos.

\section{Referencias}

Aberastury, A. y Knobel, M. (2010). La adolescencia normal. Un enfoque psicoanalítico. México: Paidós.

Carrera, B. y Mazzarella, C. (2001). Vygotsky: enfoque sociocultural. Educere, 5(13). Recuperado de https://www.redalyc.org/articulo.oa?id=356/35601309.

Castillo, S. y Cabrerizo, J. (2011). Evaluación de la intervención socioeducativa. Agentes, ámbitos y proyectos. España: Pearson Educación.

Centro Nacional de Equidad de Género y Salud Reproductiva. (2016). Modelo de atención integral en salud sexual y reproductiva para adolescentes. Ciudad de México, México: Secretaría de Salud. Recuperado de https://data.miraquetemiro.org/sites/default/files/documentos/MAISSRA\%202017.p df

Coleman, J. C. y Hendry, L. B. (2003). Psicología de la adolescencia. Londres, Inglaterra: Ediciones Morata.

Consejo de Investigación y Evaluación de la Política Social [CIEPS]. (2014). Investigación: Causas y factores de la maternidad adolescente en el Estado de México, dentro del contexto del Programa de Desarrollo Social Futuro en Grande. México: Gobierno del Estado de México.

Consejo Nacional de Población [CONAPO]. (2005). Hablemos de sexualidad en la escuela secundaria. México: Consejo Nacional de Población.

Fisher, W., Byrne, D. and White, L. (1983). Emotional Barriers to Contraception. En Byrne, D. and Fisher, W. (comps.), Adolescents, Sex, and Contraception (pp. 207-239). Hillsdale, United States: Lawrence Erlbaum.

Fundación Huésped. (2012). Guía sobre salud sexual y reproductiva y diseño de proyectos para organizaciones sociales. Buenos Aires, Argentina: Fundación Huésped. Recuperado de https://www.huesped.org.ar/wp-content/uploads/2017/04/Guia-SSRy-Diseno-de-Proyectos.pdf. 


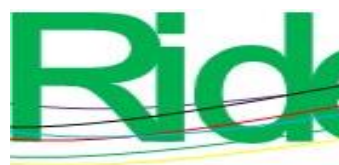

Revista Iberoamericana para la
Investigación y el Desarrollo Educativo
ISSN $2007-7467$

Gobierno de la República. (2014). Estrategia Nacional para la Prevención del Embarazo en

Adolescentes. México: Gobierno de la República. Recuperado de https://www.gob.mx/cms/uploads/attachment/file/55979/ENAPEA_0215.pdf.

Gobierno de la República. (2018). Estrategia Nacional para la Prevención del Embarazo en Adolescentes (ENAPEA). Informe 2018. México: Gobierno de la República. Recuperado de https://www.gob.mx/cms/uploads/attachment/file/425939/Informe_Ejecutivo_GIPE A_2018.pdf

Herrera, C., Campero, L., Barrera, L., González, G., Atienzo, E. E. y Estrada, F. (2018). Decir a medias: límites percibidos por los adultos para involucrarse en la prevención del embarazo adolescente en México. Nueva Antropología, 31(88), 134-154.

Krauskopf, D. (1999). El desarrollo psicológico en la adolescencia: las transformaciones en una época de cambios. Adolescencia y Salud, 1(2), 23-31.

Loredo, A., Vargas, E., Casa, A., González, J. y Gutiérrez, C. (2015). Embarazo adolescente y sus repercusiones en la diada. Revista Médica del Instituto del Seguro Social, 55(2), 223-229.

Medina, M. (2017). Formación de maestros en educación sexual. Ponencia presentada en el XIV Congreso Nacional de Investigación Educativa. San Luis Potosí, 2017. Recuperado de http://www.comie.org.mx/congreso/memoriaelectronica/v14/doc/0670.pdf

Organización de las Naciones Unidas para la Educación, la Ciencia y la Cultura [UNESCO]. (2010). Orientaciones técnicas internacionales sobre educación en sexualidad. Un enfoque basado en evidencia orientado a escuelas, docentes y educadores de la salud. Volumen I. Justificación de la educación en sexualidad. París, Francia: Organización de las Naciones Unidas para la Educación, la Ciencia y la Cultura. Recuperado de https://unesdoc.unesco.org/ark:/48223/pf0000183281_spa.

Pi, L. (4 de agosto de 2016). Padres de familia contra los libros sobre sexualidad. FOROtv. Recuperado de https://medium.com/opinion\%C3\%B3n-con-foro/padres-defamilia_contra-los-libros-sobre-sexualidad-76579bf95f4d

Rangel, J., Valerio, L., Patiño, J. y García, M. (2004). Funcionalidad familiar en la adolescente embarazada. Revista de la Facultad de Medicina UNAM, 47(1), 24-27.

Rodrigues, M. G. S., Cosentino, S. F., Rossetto, M., Maia, K. M., Pautz, M. y Silva, V. C. (2010). Talleres educativos en sexualidad del adolescente: la escuela como escenario. 


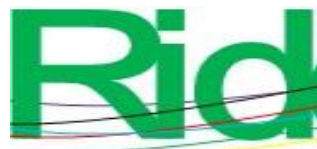

Enfermería

Global,

Recuperado

de

http://scielo.isciii.es/scielo.php?script=sci_arttext\&pid=S1695-

61412010000300009 .

Rosales, A. (2011). Sexualidad, derechos y violencia. Enfoques y conceptos para la enseñanza. México: Polvo de Gis

Salinas, S. (coord.) (2014). Vivencias y relatos sobre el embarazo en adolescentes: una aproximación a los factores culturales, sociales y emocionales a partir de un estudio en seis países de la región. Informe final. Panamá: Plan Internacional y UNICEF.

Secretaría de Educación Pública [SEP]. (2017). Aprendizajes clave para la educación integral. Plan y programas de estudio para la educación básica. México: Secretaría de Educación Pública.

Secretaría de Salud. (2013). Programa de Acción Específico. Salud sexual y reproductiva para adolescentes 2013-2018. México: Secretaría de Salud.

Stern, C. (2004). Vulnerabilidad social y embarazo adolescente en México. Papeles de Población, 10(39), 129-158.

Yuste, B. (2017). Las nuevas formas de consumir información de los jóvenes. Revista de estudios y juventud, (108), 179- 191. 


\begin{tabular}{|c|c|}
\hline Rol de Contribución & Autor (es) \\
\hline Conceptualización & $\begin{array}{l}\text { Dafne Bastida Izaguirre (igual) Irma Alejandra Martínez } \\
\text { Galván (igual) Krystel Denise Ramos López (igual) } \\
\text { Luis Alberto Ríos Dueñas (igual) }\end{array}$ \\
\hline Metodología & $\begin{array}{l}\text { Dafne Bastida Izaguirre (igual) Irma Alejandra Martínez } \\
\text { Galván (igual) Krystel Denise Ramos López (igual) } \\
\text { Luis Alberto Ríos Dueñas (igual) Fátima Aranzazu Villanueva } \\
\text { Ramírez (igual) }\end{array}$ \\
\hline Software & No aplica \\
\hline Validación & $\begin{array}{l}\text { Dafne Bastida Izaguirre (igual) Irma Alejandra Martínez } \\
\text { Galván (igual) Krystel Denise Ramos López (igual) } \\
\text { Luis Alberto Ríos Dueñas (igual) }\end{array}$ \\
\hline Análisis Formal & $\begin{array}{l}\text { Irma Alejandra Martínez Galván (igual) Krystel Denise Ramos } \\
\text { López (igual) Luis Alberto Ríos Dueñas (igual) } \\
\text { Dafne Bastida Izaguirre (que apoya) }\end{array}$ \\
\hline Investigación & $\begin{array}{l}\text { Dafne Bastida Izaguirre (igual) Irma Alejandra Martínez } \\
\text { Galván (igual) Krystel Denise Ramos López (igual) } \\
\text { Luis Alberto Ríos Dueñas (igual) } \\
\text { Fátima Aranzazu Villanueva Ramírez (igual) }\end{array}$ \\
\hline Recursos & $\begin{array}{l}\text { Irma Alejandra Martínez Galván (igual) Krystel Denise Ramos } \\
\text { López (igual) Luis Alberto Ríos Dueñas (igual) } \\
\text { Fátima Aranzazu Villanueva Ramírez (igual) } \\
\text { Dafne Bastida Izaguirre (que apoya) }\end{array}$ \\
\hline Curación de datos & Dafne Bastida Izaguirre \\
\hline $\begin{array}{l}\text { Escritura - Preparación del } \\
\text { borrador original }\end{array}$ & $\begin{array}{l}\text { Irma Alejandra Martínez Galván (igual) Krystel Denise Ramos } \\
\text { López (igual) Luis Alberto Ríos Dueñas (igual) } \\
\text { Dafne Bastida Izaguirre (que apoya) }\end{array}$ \\
\hline $\begin{array}{l}\text { Escritura - Revisión y } \\
\text { edición }\end{array}$ & Dafne Bastida Izaguirre \\
\hline Visualización & $\begin{array}{l}\text { Dafne Bastida Izaguirre (principal) Irma Alejandra Martínez } \\
\text { Galván (que apoya) }\end{array}$ \\
\hline Supervisión & Dafne Bastida Izaguirre \\
\hline $\begin{array}{l}\text { Administración de } \\
\text { Proyectos }\end{array}$ & Dafne Bastida Izaguirre \\
\hline Adquisición de fondos & Dafne Bastida Izaguirre \\
\hline
\end{tabular}

